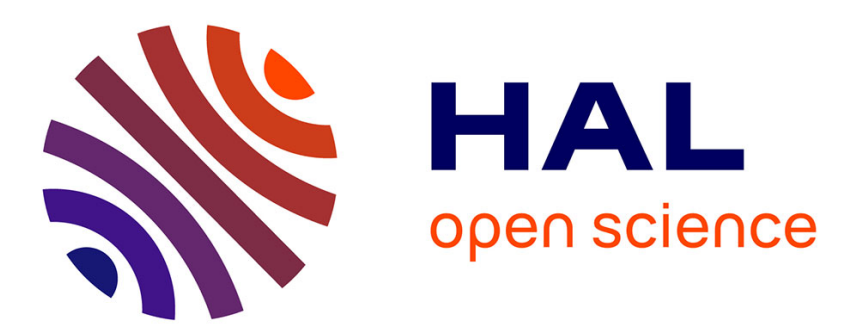

\title{
Non-Regenerative Full Distributed Space-Time Codes in Cooperative Relaying Networks
}

Le-Quang-Vinh Tran, Olivier Berder, Olivier Sentieys

\section{To cite this version:}

Le-Quang-Vinh Tran, Olivier Berder, Olivier Sentieys. Non-Regenerative Full Distributed Space-Time Codes in Cooperative Relaying Networks. Proc. of the IEEE International Wireless Communications and Networking Conference (WCNC), Mar 2011, Cancun, Mexico, France. pp.1529 - 1533. hal00746408

\section{HAL Id: hal-00746408 \\ https://hal.science/hal-00746408}

Submitted on 28 Oct 2012

HAL is a multi-disciplinary open access archive for the deposit and dissemination of scientific research documents, whether they are published or not. The documents may come from teaching and research institutions in France or abroad, or from public or private research centers.
L'archive ouverte pluridisciplinaire HAL, est destinée au dépôt et à la diffusion de documents scientifiques de niveau recherche, publiés ou non, émanant des établissements d'enseignement et de recherche français ou étrangers, des laboratoires publics ou privés. 


\title{
Non-Regenerative Full Distributed Space-Time Codes in Cooperative Relaying Networks
}

\author{
Le Quang Vinh TRAN, Olivier BERDER, Olivier SENTIEYS \\ IRISA/INRIA, University of Rennes 1 \\ 6 rue de Kerampont BP 80518 - 22305 Lannion Cedex, France \\ \{vinh.tran, oberder, sentieys\} @irisa.fr
}

\begin{abstract}
Distributed space-time codes (DSTC) are often used in cooperative relaying networks whose relays can support a single antenna due to the limited physical size. In this paper, full DSTC protocol in which there is a data exchange between relays before forwarding signals to destination is proposed to improve the performance of a cooperative relaying system. A lower bound for the average symbol error probability (ASEP) of full DSTC cooperative relaying system in a Rayleigh fading environment is provided. In the case when the Signal to Noise Ratio (SNR) of the relay-relay link is much greater than that of the source-relay link, the upper bound on ASEP of this system is also derived. From the simulations, we show that the average SNR gain of full DSTC system over DSTC system is $3.8 d B$ and the maximum SNR gain is $5 d B$ when the relay-relay distance is small and the relays are in the middle of the source and the destination. The effect of the distance between the relays shows that the performance does not degrade so much as the distance between relays is lower than a half of the source-destination distance. Moreover, we also show that when the error synchronization range is lower than 0.5 , the impact of the transmission synchronization error of the relay-destination link on the performance is not considerable.
\end{abstract}

\section{INTRODUCTION}

Recently, relay transmission has been identified as one of the core technologies that could enable robust and highreliable information transfer over challenging wireless environment. By positioning relays between source and destination, the relaying networks attract significant interest in wireless communication as it achieves the performance of Multi-Input Multi-Output (MIMO) systems through multiple relays instead of using multiple antennas at terminals. In [1], via the outage probability analysis of distributed space-time codes (DSTC) and repetition-based cooperative diversity protocol, the DSTC is shown to have full spatial diversity. In [2], a DSTC multihop system in which source and relay share their antennas to create a virtual transmit array is considered in terms of average Bit Error Rate (BER) and achievable rate. Beside, in [3], the performance of DSTC systems with one and two nonregenerative relays is given, while the performance of DSTC systems with regenerative relays is done in [4]. The DSTC protocol for wireless multi-hop networks is lately designed and the performance for communication over more than two hops is analyzed in [5].

In the present paper, a cooperative relaying system having a source, a destination and two relays all equipped with single antenna is considered. The full DSTC protocol for cooperative relaying networks in which the relays exchange data with each other and then forward the signals to destination using non-regenerative DSTC is proposed to improve the performance of the system in terms of BER. To the best of our knowledge, this is the first time the data exchange between relays in cooperative relaying networks is presented and analyzed. The lower bound expression on average symbol error probability (ASEP) of full DSTC cooperative relaying system is derived. Beside, the upper bound expression on ASEP is given in the case that the distance between relays, $d_{r r}$ is small enough in comparison with the source-destination distance $d_{s d}$ to be able to neglect the transmission errors in the data exchange of two relays. Simulation results will show that this condition is satisfied when $d_{r r}=0.1 d_{s d}$. In addition, the effects of some practical problems, such as the relative distance of relays, the distance between two relays and the transmission synchronization errors of the relay-destination link, are also considered and simulated in comparison with DSTC cooperative relaying model in which there is no data exchange between the relays.

The remainder of this paper is organized as follows. In Section II, the protocol of full DSTC cooperative relaying system is thoroughly described. Then the average error probability analysis is considered in Section III. Section IV presents the simulation results, while Section $\mathrm{V}$ proposes a discussion and conclusion.

\section{SySTEM MODEL}

The full distributed space-time coded cooperative relaying network considered in this paper includes one source, one destination and two relays which all have only one antenna. After receiving the signals from the source, the two relays exchange data with each other, then combine all the received signals using Maximum Ratio Combining (MRC) technique and finally forward them to destination using DSTC. We assume that all communications are performed over flat Rayleigh fading channel. The channel coefficients remain the same for two consecutive time intervals. Statistically, we model the channel coefficient $h_{i j}$ with $i \in\left\{s, r_{1}, r_{2}\right\}, j \in\left\{r_{1}, r_{2}, d\right\}$ and $i \neq j$ as zero mean, independent, complex Gaussian random variables with variances $\Omega_{i j}$. Similarly, we model the AWGN noise $z_{i j}[n]$ as zero mean mutually independent complex Gaussian random variable with variance $N_{i j}$. Without loss of generality we assume that $N_{i j}=1, \forall i, j$. 


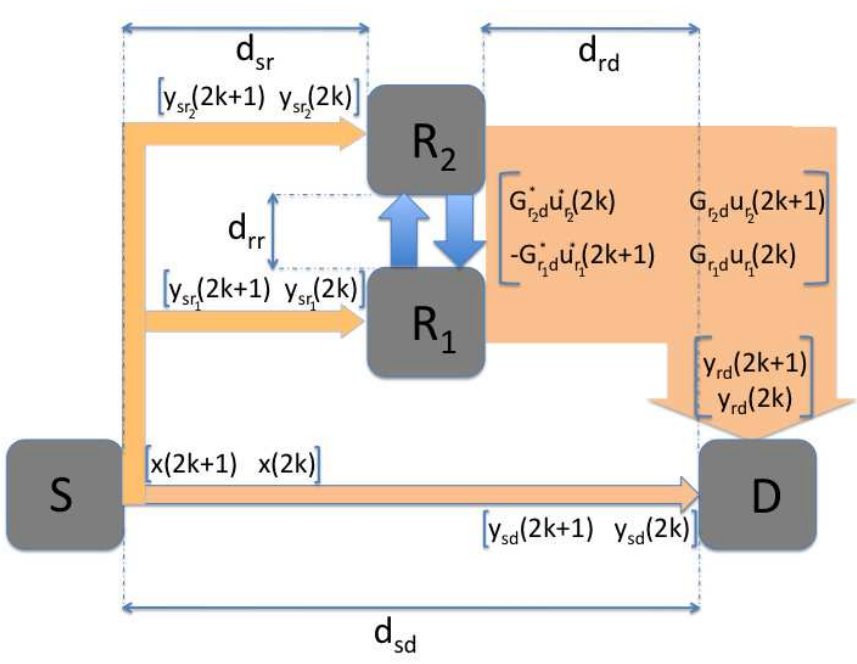

Fig. 1. Full Distributed Space Time Code Cooperative Relaying Model

The transmission protocol of this model can be described as below. Firstly, the source transmits signals to the relays and the destination at the same time. The received signals at relays and destination can be represented as $y_{s r 1}, y_{s r 2}$ and $y_{s d}$ respectively

$$
y_{s j}(n)=\sqrt{\epsilon_{s}} h_{s j} x[n]+z_{s j}[n], \quad j \in\left\{r_{1}, r_{2}, d\right\}
$$

where $x[n]$ is the source transmitted signal and $\epsilon_{s}$ is the transmitted source power.

Secondly, the two relays exchange their data with each other. The received signals at relay $R_{1}$ from relay $R_{2}$ and vice versa, respectively symbolized as $y_{r_{2} r_{1}}$ and $y_{r_{1} r_{2}}$, are given by

$$
y_{i j}(n)=h_{i j} G_{s i} y_{s i}[n]+z_{i j}[n], \quad i, j \in\left\{r_{1}, r_{2}\right\}, i \neq j
$$

where $G_{s i}$ is the maximum gain [6] which satisfies the output power constraint of relay $i$ and can be chosen as

$$
G_{s i}=\sqrt{\frac{\epsilon_{i}}{\epsilon_{s}\left|h_{s i}\right|^{2}+1}}, \quad i \in\left\{r_{1}, r_{2}\right\}
$$

with $\epsilon_{i}$ the transmitted power of relay $i$.

Thirdly, each relay uses a Maximum Ratio Combiner to combine the signals received from source and from the other relay

$$
u_{j}(n)=h_{s j}^{*} y_{s j}[n]+\frac{h_{i j}^{*} G_{s i}^{*} h_{s i}^{*}}{\left|h_{i j}\right|^{2}\left|G_{s i}\right|^{2}+1} y_{i j}[n]
$$

where $i, j \in\left\{r_{1}, r_{2}\right\}$ and $i \neq j$.

Finally, the two relays use non-regenerative DSTC protocol to transmit simultaneously the Alamouti re-encoded signals $\boldsymbol{U}=\left[\begin{array}{cc}G_{r_{1} d} u_{r_{1}}[2 k] & G_{r_{2} d} u_{r_{2}}[2 k+1] \\ -G_{r_{1} d}^{*} u_{r_{1}}^{*}[2 k+1] & G_{r_{2} d}^{*} u_{r_{2}}^{*}[2 k]\end{array}\right]$ to the destination. The signals received from the relays at destination, $y_{\boldsymbol{r d}}=$ $\left[y_{r d}[2 k] \quad y_{r d}[2 k+1]\right]^{T}$, are

$$
y_{r d}=U h_{r d}+z_{r d}
$$

where $\boldsymbol{h}_{\boldsymbol{r d}}=\left[h_{r d}[2 k] \quad h_{r d}[2 k+1]\right]^{T}$ is the Rayleigh channel coefficient vector of the relay-destination link and $z_{r d}=$ $\left[z_{r d}[2 k] \quad z_{r d}[2 k+1]\right]^{T}$ is the AWGN noise vector of the relaydestination link.

Similarly, to prevent the saturation, the maximum gain $G_{j d}$ at relay $j$ is chosen as

$$
G_{j d}=\sqrt{\frac{\epsilon_{j}}{\epsilon_{s}\left|a_{j}\right|^{2}+a_{j}}}, \quad j \in\left\{r_{1}, r_{2}\right\}
$$

where $a_{j}=\left|h_{s j}\right|^{2}+\frac{\left|h_{i j} G_{s i} h_{s i}\right|^{2}}{\left|h_{i j} G_{s i}\right|^{2}+1} \quad i, j \in\left\{r_{1}, r_{2}\right\}, i \neq j$ and $\epsilon_{j}$ is the transmitted power of relay $j$.

At the destination, the Alamouti receiver is used to process the signals received from $R_{1}$ and $R_{2}$. Then, the output of the Alamouti receiver is combined with $\left[y_{s d}[2 k] \quad y_{s d}[2 k+1]\right]^{T}$ using Maximum Ratio Combining technique.

\section{Average Symbol Error Probability AnAlysis}

With the fading realizations $h_{i j}, i \in\left\{s, r_{1}, r_{2}\right\}, j \in\left\{r_{1}, r_{2}, d\right\}$ and $i \neq j$, the Signal to Noise Ratio at terminal $j$ after receiving signals from terminal $i$ is found as $\gamma_{i j}=\epsilon_{i}\left|h_{i j}\right|^{2} / N_{i j}$ which has exponential distribution with the mean $\overline{\gamma_{i j}}=\frac{\epsilon_{i} \Omega_{i j}}{N_{i j}}$. The density probability function of $\gamma_{i j}$ is represented by

$$
f_{\gamma_{i j}}(\gamma)=\frac{1}{\overline{\gamma_{i j}}} e^{-\frac{\gamma}{\gamma_{i j}}}
$$

With the choice of $G_{s i}, \quad i \in\left\{r_{1}, r_{2}\right\}$ in (3), the post-detection $\mathrm{SNR}$ at relay $i$ can be referred as

$$
\gamma_{i}=\gamma_{s i}+\frac{\gamma_{s j} \gamma_{j i}}{\gamma_{s j}+\gamma_{j i}+1} \quad i, j \in\left\{r_{1}, r_{2}\right\}, i \neq j
$$

Similarly, the post-detection SNR at destination is

$$
\gamma_{d}=\gamma_{s d}+\epsilon_{s} \frac{\sum_{j \in\left\{r_{1}, r_{2}\right\}}\left|h_{j d} G_{j d} a_{j}\right|^{2}}{\sum_{j \in\left\{r_{1}, r_{2}\right\}}\left|h_{j d} G_{j d}\right|^{2} a_{j}+1}
$$

Because the distribution of $\gamma_{d}$ is not easy to find, we would like to find and use the distribution of $\gamma^{L}$ and $\gamma^{U}$ to upper bound and lower bound the ASEP of full DSTC cooperative relaying system. We find that

$$
\gamma^{L}<\gamma_{d}<\gamma^{U}
$$

where $\gamma^{L}$ is the lower bound of $\gamma_{d}$ only when $\gamma_{i j} \gg \gamma_{s j}, i, j \in$ $\left\{r_{1}, r_{2}\right\}, i \neq j$

$$
\begin{aligned}
\gamma^{L} & =\gamma_{s d}+\frac{\gamma_{s r_{1}} \gamma_{r_{1} d}}{\gamma_{s r_{1}}+\gamma_{r_{1} d}+1}+\frac{\gamma_{s r_{2}} \gamma_{r_{2} d}}{\gamma_{s r_{2}}+\gamma_{r_{2} d}+1} \\
& =\gamma_{s d}+\gamma_{s r_{1} d}^{L}+\gamma_{s r_{2} d}^{L}
\end{aligned}
$$

and $\gamma^{U}$ is the upper bound of $\gamma_{d}$

$$
\begin{aligned}
\gamma^{U} & =\gamma_{s d}+\frac{\left(\gamma_{s r_{1}}+\gamma_{s r_{2}}\right) \gamma_{r_{1} d}}{\gamma_{s r_{1}}+\gamma_{r_{1} d}+\gamma_{s r_{2}}+1}+\frac{\left(\gamma_{s r_{2}}+\gamma_{s r_{1}}\right) \gamma_{r_{2} d}}{\gamma_{s r_{1}}+\gamma_{s r_{2}}+\gamma_{r_{2} d}+1} \\
& =\gamma_{s d}+\gamma_{s r_{1} d}^{U}+\gamma_{s r_{2} d}^{U}
\end{aligned}
$$

From (10), we find that the lower bound on ASEP of full DSTC cooperative relaying system is $P_{\gamma_{d}}^{L}=P_{\gamma U}$ and the upper bound on ASEP of full DSTC cooperative relaying system is $P_{\gamma_{d}}^{U}=P_{\gamma^{L}}$. We have

$$
P_{\gamma_{d}}^{L}=P_{\gamma^{U}}<P_{\gamma_{d}}<P_{\gamma^{L}}=P_{\gamma_{d}}^{U}
$$

In order to find the lower bound for the performance of full DSTC cooperative relaying system, we first derive the moment generating functions (MGFs) of $\gamma_{s i d}^{U}, i \in\left\{r_{1}, r_{2}\right\}$ which is proved in Appendix A.

$$
\begin{aligned}
M_{\gamma_{\text {sid }}^{U}}(s)= & e^{\alpha_{i} / 2}\left[\frac{2}{p_{i} \sqrt{p_{i}}}\left(\left(\frac{p_{i}}{\overline{\gamma_{i d}}}-\sigma_{i}+\frac{\sigma_{i}}{2 \overline{\gamma_{i d}}}\right) \frac{\delta J_{0}}{\delta \beta}+\left(\frac{\sigma_{i}}{\overline{\gamma_{i d}}}+2\right) \frac{\delta^{2} J_{0}}{\delta \alpha \delta \beta}\right)\right. \\
& \left.+\frac{2}{p_{i}^{2}}\left(\left(\frac{2 p_{i}}{\overline{\gamma_{i d}}}+\sigma_{i}\right) \frac{\delta^{2} J_{0}}{\delta \alpha^{2}}+\frac{p_{i}}{\overline{\gamma_{i d}}} \frac{\delta J_{0}}{\delta \alpha}-\frac{\sigma_{i}}{4} J_{0}\right)\right]
\end{aligned}
$$

where $t_{0}=\overline{\gamma_{i d}}, \sigma=\sigma_{i}=\overline{\gamma_{i d}}+\overline{\gamma_{s i}}, p=p_{i}=\overline{\gamma_{i d} \gamma_{s i}}$, and $\alpha_{i}=$ $\left(\sigma_{i}-p_{i} s\right) / p_{i}$. The formula of $J_{0}, \frac{\delta J_{0}}{\delta \alpha}, \frac{\delta J_{0}}{\delta \beta}, \frac{\delta^{2} J_{0}}{\delta \alpha^{2}}$ and $\frac{\delta^{2} J_{0}}{\delta \alpha \delta \beta}$ can be found in Appendix A. Note that with the assumption $\overline{\gamma_{s r}}=$ 
$\overline{\gamma_{s r_{1}}}=\overline{\gamma_{s r_{2}}}$, the random variable $\gamma_{s r}=\gamma_{s r_{1}}+\gamma_{s r_{2}}$ will have the probability density function $f_{\gamma_{s r}}(\gamma)=\frac{1}{\bar{\gamma}_{s r}} 2 e^{-\frac{\gamma}{\gamma_{s r}}}$.

Then, the lower bound ASEP of full DSTC cooperative relaying system, $P_{\gamma_{d}}^{L}$, for BPSK modulation can be derived based on MGF-based approach of [7, Eq. 9.12]

$$
P_{\gamma_{d}}^{L}=\frac{1}{\pi} \int_{0}^{\pi / 2} \frac{\prod_{i \in\left\{r_{1}, r_{2}\right\}} M_{\gamma_{s i d}^{U}}\left(-\frac{g_{B P S K}}{\sin ^{2} \theta}\right)}{1+\frac{g_{B P S K} \overline{\gamma_{s d}}}{\sin ^{2} \theta}} d \theta
$$

where $g_{B P S K}=1$ [7] and $M_{\gamma_{s d}}=\left(1+\frac{g_{B P S K} \overline{\gamma_{s d}}}{\sin ^{2} \theta}\right)^{-1}$ [7, Tab 9.1].

Similarly, the upper bound ASEP of full DSTC cooperative relaying system, $P_{\gamma_{d}}^{U}$, can be written

$$
P_{\gamma_{d}}^{U}=\frac{1}{\pi} \int_{0}^{\pi / 2} \frac{\prod_{i \in\left\{r_{1}, r_{2}\right\}} M_{\gamma_{s i d}^{L}}\left(-\frac{g_{B P S K}}{\sin ^{2} \theta}\right)}{1+\frac{g_{B P S K} \overline{\gamma_{s d}}}{\sin ^{2} \theta}} d \theta
$$

Fortunately, the moment generating functions of $\gamma_{\text {sid }}^{L}, i \in$ $\left\{r_{1}, r_{2}\right\}$ are already found in [8, Eq. 13]

$$
M_{\gamma_{\text {sid }}^{L}}(s)=\frac{2}{p_{i}} e^{\alpha_{i} / 2}\left[-2 \frac{\delta J_{0}}{\delta \alpha}-\frac{\sigma_{i}}{p_{i}} \frac{\delta J_{0}}{\delta \beta}\right]
$$

where $\sigma=\overline{\gamma_{i d}}+\overline{\gamma_{s i}}, p=\overline{\gamma_{i d} \gamma_{s i}}$ and $\alpha_{i}=\left(\sigma_{i}-p_{i} s\right) / p_{i}$. The formula of $\frac{\delta J_{0}}{\delta \alpha}$ and $\frac{\delta J_{0}}{\delta \beta}$ is given in Appendix A.

On the other hand, for DSTC cooperative relaying model, where there is no data exchange between the two relays, the post-detection SNR at destination is found as

$$
\gamma_{d}^{\prime}=\gamma_{s d}+\epsilon_{s} \frac{\sum_{j \in\left\{r_{1}, r_{2}\right\}}\left|h_{j d} G_{j d}^{\prime} a_{j}^{\prime}\right|^{2}}{\sum_{j \in\left\{r_{1}, r_{2}\right\}}\left|h_{j d} G^{\prime}{ }_{j d}\right|^{2}+1}
$$

where $a_{j}^{\prime}=h_{s j}$ and $G_{j d}^{\prime}=\frac{h_{s j}^{*}}{\left|h_{s j}\right|} \sqrt{\frac{\epsilon_{j}}{\epsilon_{s}\left|h_{s j}\right|^{2}+1}}$.

We find that $\gamma_{d}^{\prime}<\gamma^{L}$ so $P_{\gamma_{d}}^{U}=P_{\gamma^{L}}$ is also the lower bound on ASEP of DSTC cooperative relaying system.

\section{Simulation RESUlts}

For the convenience of the symbolization, we denote $r$ the relative distance of relays which is defined by the ratio of the source-relay distance $d_{s r}$ and the source-destination distance $d_{s d}$ and let $r r$ be the relative inter-distance of relays which is defined by the ratio of the relay-relay distance $d_{r r}$ and the source-destination distance $d_{s d}$. Besides, the power is equally allocated among transmitters, i.e. $\epsilon_{i j}=\epsilon$, and the BPSK modulation is selected.

\section{A. Bounds for full DSTC cooperative relaying system}

For the comparison between the analytic results and simulation results, we first consider the special case of symmetric network in which the fading variances are identical, e.g, $\Omega_{i j}=1 \forall i, j$. In this simulation, the condition that $\gamma_{i j} \gg \gamma_{s j}$, $i, j \in\left\{r_{1}, r_{2}\right\}, i \neq j$ is made sure. We can see from Fig. 2 that the performance simulation of full DSTC cooperative relaying system is exactly in the middle of the upper bound on ASEP, $P_{\gamma_{d}}^{U}$ and lower bound on ASEP, $P_{\gamma_{d}}^{L}$. Moreover, from the simulation results, $P_{\gamma_{d}}^{U}$ is shown to be the lower bound on ASEP of DSTC cooperative relaying system.

\section{B. Effect of the distance between two relays}

For the following subsections, the effect of geometry is taken into account. Using a common model for path-loss, we

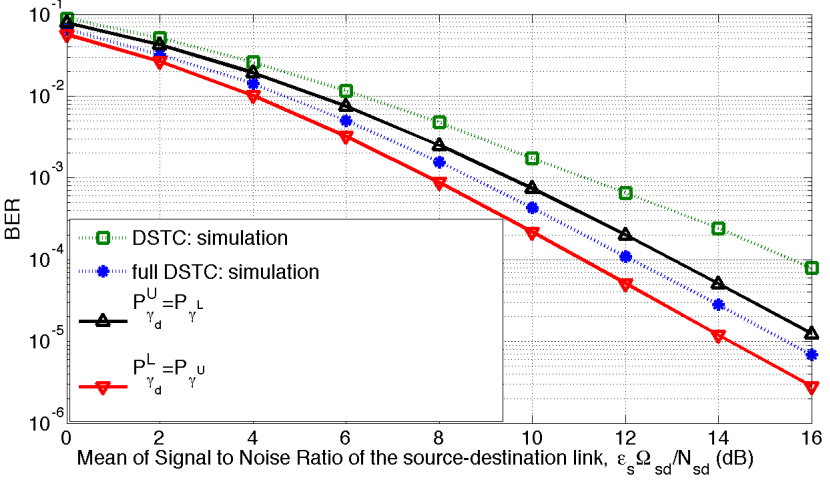

Fig. 2. Upper bound and lower bound of full DSTC cooperative relaying system

set $\Omega_{i j} \propto d_{i j}^{-\alpha}$ where $d_{i j}$ is the distance between terminal $i$ and terminal $j$, and $\alpha$ is the path-loss exponent. In our work, $\alpha=2$ is used. Fig. 3 shows the effects on BER of full cooperative relaying system in the case that $r=0.5$ versus the change of $r r$. We see that when $r r=0.1$, the performance of the system is the same as the ideal case, $r r=0$, where there is no errors in the inter-transmission between two relays. In fact, this case represents the most popular one in reality, for example: as $d_{s r}=100 \mathrm{~m}$, we can choose without difficulty two relays so that $d_{r r}=10 \mathrm{~m}$ because normally, the distance between two relays is small. Moreover, the performance of the system is not much degraded until $r r=0.5$. Otherwise, when $r r \rightarrow \infty$, i.e. $r r=1000$ in Fig. 3, the performance of full DSTC cooperative relaying system logically goes to that of DSTC cooperative relaying system. Because, if the SNR of the relay-relay link is much worse than that of the source-relay link, the data exchange between two relays does not bring any gain in comparison with DSTC cooperative relaying system. So, the results here can illustrate the accuracy of the simulations.

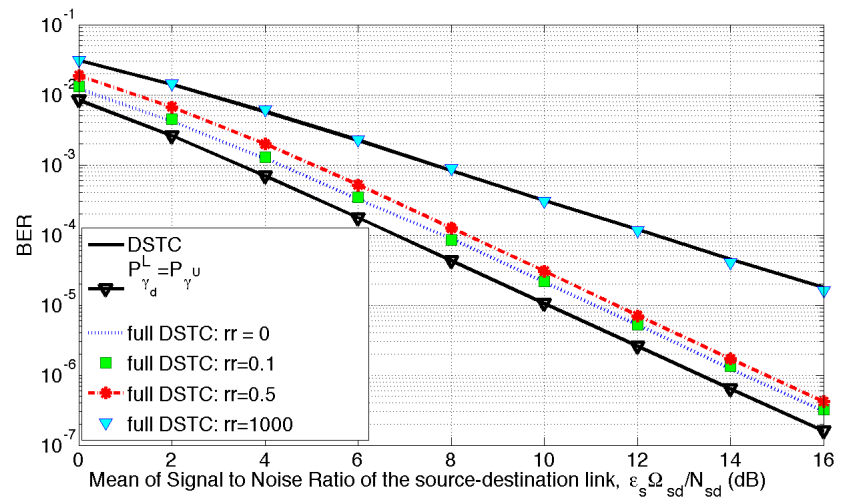

Fig. 3. Effect of the distance between two relays with the relative distance of relay, $r=0.5$

\section{Effect of the relative distance of relays}

In this subsection, we take the effect of the relative distance of relay into account, as illustrated by Fig. 4, in the case 


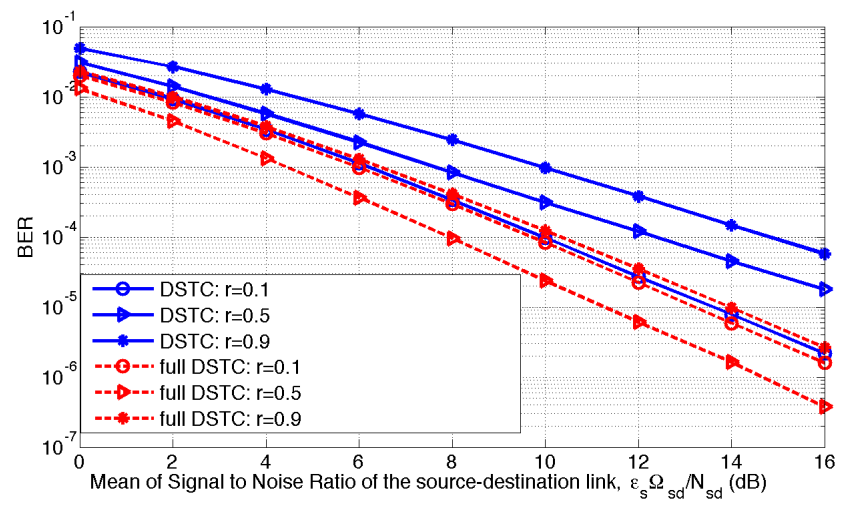

Fig. 4. Effect of the relative distance of relays on the performance of full DSTC and DSTC cooperative relaying system in the case of $r r=0.1$

of $r r=0.1$. When the relative distance of relay is small, i.e. $r=0.1$, the performance gain of full DSTC cooperative relaying system over DSTC cooperative relaying system is not considerable. However, when the relative distance of relay increases, that performance gain is much more significant. Fig. 5 shows the SNR gain of full DSTC cooperative relaying system over DSTC cooperative relaying system at $B E R=10^{-5}$ and $r r=0.1$ as a function of the relative distance of relay, $r$. The reference performance is the performance of DSTC system in the case that $r=0.9$. The average SNR gain of full DSTC cooperative relaying system over DSTC cooperative relaying system is $3.8 d B$. The maximum SNR gain of full DSTC cooperative relaying system obtained is $5 d B$ in comparison with DSTC cooperative relaying system when $r=0.5$.

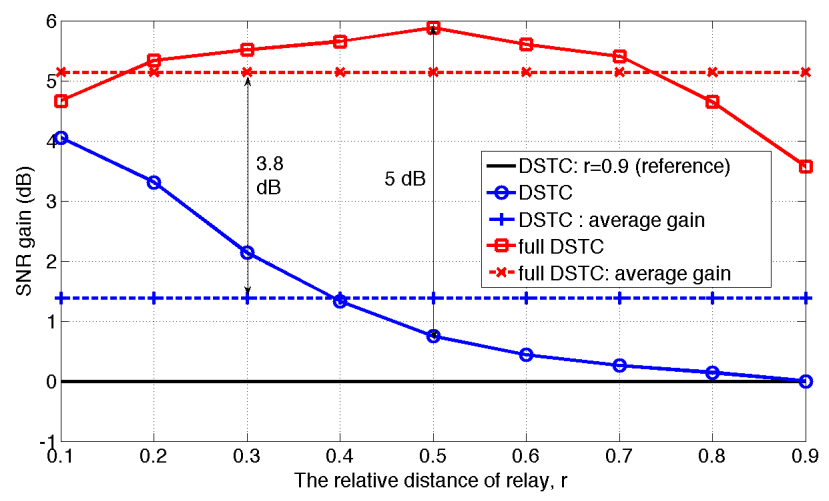

Fig. 5. The SNR gain of full DSTC cooperative relaying system over DSTC cooperative relaying system at $B E R=10^{-5}$ and $r r=0.1$ with respect to the relative distance of relay, $r$.

\section{Effect of transmission synchronization error}

As we know, a cooperative system suffers a large impact from the lack of synchronization between distributed nodes. It is no exception for full DSTC cooperative relaying system and DSTC cooperative relaying system. Here, we face with the transmission synchronization errors of the relaydestination link. Using the same synchronization error model

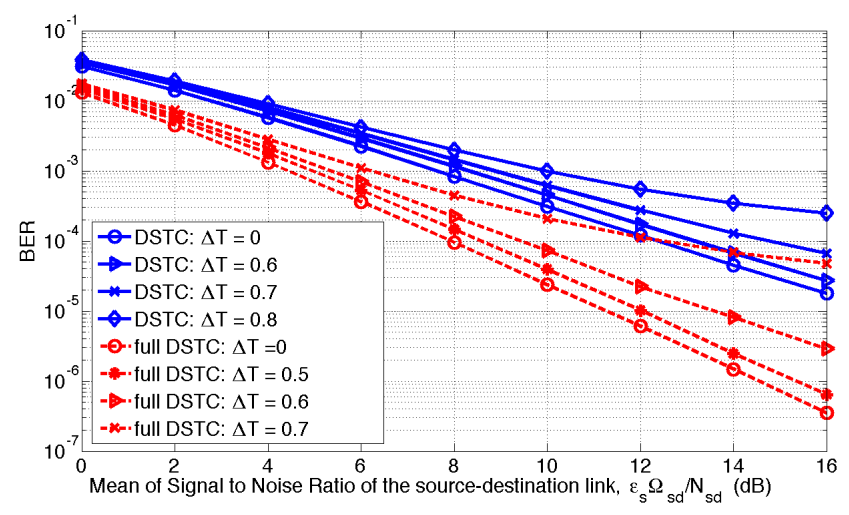

Fig. 6. Effect of transmission synchronization error on full DSTC cooperative relaying system and DSTC cooperative relaying system at the case of $r=0.5$ and $r r=0.1$

as [9] in which the interference inter-symbol (ISI) is just created by the four nearest neighbor symbols, the effect of transmission synchronization error on full DSTC cooperative relaying system and DSTC cooperative relaying system at the case of $r=0.5$ and $r r=0.1$ is considered in Fig. 6. In this simulation, the synchronization error $\delta_{k}$, [9], is considered to have a uniform distribution in $[-\Delta T / 2, \Delta T / 2]$ with $\Delta T$ the error synchronization range. We see that the performance of two models is not much degraded until $\Delta T=0.5$. For full DSTC cooperative relaying model, the performance becomes saturated at $\Delta T=0.7$ while the performance of DSTC cooperative relaying model goes to saturation at $\Delta T=0.8$.

\section{DISCUSSION AND CONCLUSION}

In this paper, a full distributed space-time coded protocol for cooperative relaying model is proposed. High SNR gain of full DSTC cooperative relaying system over DSTC cooperative relaying system, as we illustrated in the above section, comes at a price of decreasing bandwidth efficiency because of the data exchange between two relays. Full DSTC protocol is, therefore, suitable for applications of wireless sensor networks (WSNs) where the most important constraint is not spectral efficiency, but energy efficiency. On the other hand, as compared to DSTC cooperative relaying system, full DSTC one requires more complicated signal processing. However, it is easier to synchronize the DSTC transmission from relays to destination in full DSTC cooperative relaying system than in DSTC cooperative relaying system by taking advantage of the data exchange between two relays. Due to the data exchange between two relays, we can add some information on the synchronization at the Medium Access Control (MAC) layer to synchronize the data transfer of two relays to destination.

In conclusion, this paper presents the advantages of full DSTC protocol in comparison with conventional DSTC protocol in terms of BER performance. The lower bound and upper bound on ASEP of full DSTC cooperative relaying system is also derived. We showed that the average SNR gain of full DSTC cooperative relaying system over DSTC cooperative relaying system is $3.8 d B$ and the maximum SNR gain is $5 d B$ 
when the relay-relay distance is small and the relays are in the middle of the source and the destination. In addition, the performance of full DSTC cooperative relaying system is not degraded so much when the relative inter-distance of relays, $r r$, is below 0.5 . Finally, the impact of transmission synchronization errors of the relay-destination link is shown to be not considerable as the error synchronization range is below $50 \%$ of the symbol duration.

\section{APPENDiX A \\ PROOF OF (14)}

To obtain the lower bound ASEP using MGF-based approach, we need to find the MGF of $\Gamma(v, t)=\frac{v t}{v+t+1}$, where $v$ and $t$ are two independent random variables with the probability density function (PDF)

$$
\begin{aligned}
f_{v}(v) & =\frac{1}{v_{0}^{2}} v e^{-\frac{v}{v_{0}}} \\
f_{t}(t) & =\frac{1}{t_{0}} e^{-\frac{t}{t_{0}}}
\end{aligned}
$$

The cumulative distribution function (CDF) of $\Gamma(v, t)$ is found for $\gamma>0$ based on [10]

$$
\begin{aligned}
F_{\Gamma}(\gamma)=1 & -\frac{2 \sqrt{\gamma^{2}+\gamma}}{\sqrt{p}}\left(1+\frac{\gamma}{t_{0}}\right) e^{-\frac{\sigma}{p} \gamma} K_{1}\left(\frac{2}{\sqrt{p}} \sqrt{\gamma^{2}+\gamma}\right) \\
& -\frac{2}{p}\left(\gamma^{2}+\gamma\right) e^{-\frac{\sigma}{p} \gamma} K_{0}\left(\frac{2}{\sqrt{p}} \sqrt{\gamma^{2}+\gamma}\right)
\end{aligned}
$$

where $\sigma=t_{0}+v_{0}, p=t_{0} v_{0}$, and $K_{l}($.$) denotes the modified$ Bessel function of the second kind with order $l$.

The PDF of $\Gamma(v, t)$ is found by taking the derivative of $F_{\Gamma}(\gamma)$ with respect to $\gamma$ and using [11, Eq. 9.6.26]

$$
\begin{aligned}
f_{\Gamma}(\gamma)= & \frac{2}{p \sqrt{p}}\left(-\left(\frac{p}{t_{0}}-\sigma-1\right)+\left(\frac{\sigma}{t_{0}}+2\right) \gamma\right) e^{-\frac{\sigma}{p} \gamma} K_{1}\left(\frac{2}{\sqrt{p}} \sqrt{\gamma^{2}+\gamma}\right) \\
& +\frac{2}{p^{2}}\left(\left(\frac{2 p}{t_{0}}+\sigma\right) \gamma^{2}+\left(\frac{p}{t_{0}}+\sigma\right) \gamma\right) e^{-\frac{\sigma}{p} \gamma} K_{0}\left(\frac{2}{\sqrt{p}} \sqrt{\gamma^{2}+\gamma}\right)
\end{aligned}
$$

The moment generating function (MGF) is derived by using its definition and using the change of variable $\gamma \rightarrow \gamma_{1}-1 / 2$

$$
\begin{aligned}
M_{\Gamma}(s)= & e^{\alpha / 2}\left[\frac{2}{p \sqrt{p}}\left(\left(\frac{p}{t_{0}}-\sigma+\frac{\sigma}{2 t_{0}}\right) \frac{\delta J_{0}}{\delta \beta}+\left(\frac{\sigma}{t_{0}}+2\right) \frac{\delta^{2} J_{0}}{\delta \alpha \delta \beta}\right)\right. \\
& \left.+\frac{2}{p^{2}}\left(\left(\frac{2 p}{t_{0}}+\sigma\right) \frac{\delta^{2} J_{0}}{\delta \alpha^{2}}+\frac{p}{t_{0}} \frac{\delta J_{0}}{\delta \alpha}-\frac{\sigma}{4} J_{0}\right)\right]
\end{aligned}
$$

where $\alpha=(\sigma-p s) / p, \beta=2 / \sqrt{p}$ and

$$
\begin{aligned}
J_{0} & =\int_{1 / 2}^{\infty} e^{-\alpha \gamma_{1}} K_{0}\left(\beta \sqrt{\gamma_{1}^{2}-(1 / 2)^{2}}\right) \\
& =\frac{1}{2 c}\left[e^{-\frac{c}{2}} E_{1}\left(\frac{\alpha-c}{2}\right)-e^{\frac{c}{2}} E_{1}\left(\frac{\alpha+c}{2}\right)\right]
\end{aligned}
$$

with $c=\sqrt{\alpha^{2}-\beta^{2}}, \operatorname{Re}\{s\}<\sigma / p+2 / \sqrt{(p)}$ and $E_{1}($.$) the$ exponential integral function defined as [11, Eq. 5.1.1]. Note that $J_{0}$ is derived by using the integrand in [12, Eq. 646].

Taking the derivative of $J_{0}$ with respect to $\alpha$ or/and $\beta$ with the respective order, we can get

$$
\begin{aligned}
\frac{\delta J_{0}}{\delta \alpha}= & -\frac{\alpha}{4 c^{3}}(2+c) e^{-\frac{c}{2}} E_{1}\left(\frac{\alpha-c}{2}\right)+\frac{e^{\frac{-\alpha}{2}}}{c^{2}} \\
& +\frac{\alpha}{4 c^{3}}(2-c) e^{\frac{c}{2}} E_{1}\left(\frac{\alpha+c}{2}\right)
\end{aligned}
$$

$$
\begin{gathered}
\frac{\delta J_{0}}{\delta \beta}=\frac{\beta}{4 c^{3}}(2+c) e^{-\frac{c}{2}} E_{1}\left(\frac{\alpha-c}{2}\right)-\frac{\alpha}{\beta c^{2}} e^{\frac{-\alpha}{2}} \\
-\frac{\beta}{4 c^{3}}(2-c) e^{\frac{c}{2}} E_{1}\left(\frac{\alpha+c}{2}\right) \\
\frac{\delta^{2} J_{0}}{\delta \alpha^{2}}=\frac{\alpha^{2}}{c^{5}}\left(1+\frac{c}{2}+\frac{\beta^{2}}{2 \alpha^{2}}+\frac{\beta^{2} c}{4 \alpha^{2}}+\frac{c^{2}}{8}\right) e^{-\frac{c}{2}} E_{1}\left(\frac{\alpha-c}{2}\right) \\
+\frac{\alpha^{2}}{c^{5}}\left(-1+\frac{c}{2}-\frac{\beta^{2}}{2 \alpha^{2}}+\frac{\beta^{2} c}{4 \alpha^{2}}-\frac{c^{2}}{8}\right) e^{\frac{c}{2}} E_{1}\left(\frac{\alpha+c}{2}\right) \\
+\frac{1}{c^{4}}\left(-\frac{c^{2}}{2}-3 \alpha\right) e^{\frac{-\alpha}{2}} \\
\frac{\delta^{2} J_{0}}{\delta \alpha \delta \beta}=\frac{1}{c^{5}}\left(-\frac{3}{2} \alpha \beta-\frac{3}{4} \alpha \beta c-\frac{1}{8} \alpha \beta c^{2}\right) e^{-\frac{c}{2}} E_{1}\left(\frac{\alpha-c}{2}\right) \\
+\frac{1}{c^{5}}\left(\frac{3}{2} \alpha \beta-\frac{3}{4} \alpha \beta c+\frac{1}{8} \alpha \beta c^{2}\right) e^{\frac{c}{2}} E_{1}\left(\frac{\alpha+c}{2}\right) \\
+\frac{1}{c^{4}}\left(\frac{\alpha^{2}}{\beta}+\frac{\alpha c^{2}}{2 \beta}+2 \beta\right) e^{\frac{-\alpha}{2}}
\end{gathered}
$$

\section{REFERENCES}

[1] J. N. Laneman and G. W. Wornell, "Distributed space-time-coded protocols for exploiting cooperative diversity in wireless networks," IEEE Transaction on Information Theory, vol. 49, no. 10, pp. 24152425, Oct. 2003.

[2] S. Barbarossa and G. Scutari, "Distributed space-time coding for multihop networks," IEEE International Conference on Acoustics, Speech and Signal Processing (ICASSP04), pp. 501-504, May 2004.

[3] P. A. Anghel and M. Kaveh, "On the performance of distributed spacetime coding systems with one and two non-regenerative relays," IEEE Transactions on Wireless Communications, no. 3, pp. 682-692, Mar. 2006.

[4] P. A. Anghel, G. Leus, and M. Kaveh, "Distributed space-time cooperative systems with regenerative relays," IEEE Transactions on Wireless Communications, no. 11, pp. 3130-3140, Nov. 2006.

[5] M. Vajapeyjan and U. Mitra, "Performance analysis of distributed spacetime coded protocols for wireless multi-hop communications," IEEE Transactions on Wireless Communications, no. 1, pp. 122-133, Jan. 2010.

[6] J. N. Laneman and G. W. Wornell, "Energy-efficient antenna sharing and relaying for wireless networks," Wireless Communications and Networking Conference, vol. 1, pp. 7-12, 2000.

[7] M. K. Simon and M. S. Alouini, Digital Communication over Fading Channels. New York, NY: John Wiley \& Sons, Inc., 2000.

[8] P. A. Anghel, G. Leus, and M. Kaveh, "Multi-user space-time coding in cooperative networks," IEEE International Conference on Acoustics, Speech and Signal Processing (ICASSP03), pp. 73-76, Apr. 2003.

[9] T. D. Nguyen, O. Berder, and O. Sentieys, "Impact of transmission synchronization error and cooperative reception techniques on the performance of cooperative mimo systems," IEEE International Communication Conference (ICC), China, pp. 4601-4605, May 2008.

[10] V. Emanian, P. A. Anghel, and M. Kaveh, "Multi-user spatial diversity in a shadow-fading environment," Vehicular Technology Conference, pp. 573-576, 2002.

[11] M. Abramowitz and I. A. Stegun, Handbook of Mathematical Functions with Formulas, Graphs, and Mathematical Tables. New York, NY: Dover Publications, ninth ed., 1970.

[12] I. S. Rappaport and I. M. Ryzhik, Table of Integrals, Series and Products. San Diego, CA: Academic Press, fifth ed., 1994. 